Rev. Elev. Méd. vét. Pays trop., 1973, 26 (1) : 105-116.

\title{
Embouche intensive de zébus Peulh sénégalais à base de paille de riz
}

\author{
par H. CALVET (*) et J. VALENZA (**) \\ [avec la collaboration technique de A.M. WANE (**)]
}

\begin{abstract}
RESUME
Les auteurs ont étudié la valeur de la paille de riz comme aliment de base pour l'embouche intensive de zébus Peulh sénégalais.

C'est un fourrage bien appété qui voit ses qualités nutritionnelles nettement augmentées par l'adjonction de mélasse.

La formule qui correspond au mieux aux conditions économiques de l'embouche à la paille de riz réside dans l'addition de mélasse et d'un supplément azoté comportant de l'urée.

Les animaux entiers ont présenté un gain de poids supérieur à celui des animaux castrés.
\end{abstract}

\section{INTRODUCTION}

L'expérimentation, objet de ce travail s'inscrit dans le cadre du programme général, exécuté par l'Institut pour déterminer les conditions techniques et économiques de l'embouche intensive des bovins sénégalais, par utilisation des sous-produits de l'agriculture.

Les essais antérieurs, poursuivis depuis 1969 , ont mis en œuvre des rations ayant la coque d'arachide mélassée comme élément de base.

L'installation d'entreprises d'embouche utilisant ce type d'alimentation se situe donc logiquement à proximité des usines de décorticage de l'arachide et tout spécialement dans la presqu'île du Cap Vert.

Mais dans les zones rizicoles du Sénégal, la région du Fleuve, la Casamance et le Sine Saloum, la production du paddy laisse des quantités importantes de sous-produits, la paille

(*) Laboratoire National de l'Elevage, B.P. 2057 Dakar-Hann, Sénégal.

(**) Laboratoire National de l'Elevage, Sangalkam, de riz en particulier, qui devraient pouvoir être valorisés par la production de viande.

Les résultats d'un premier essai utilisant la paille de riz comme fourrage ont déjà été rapportés en 1970 . Cet article constitue une étude complémentaire visant à préciser les techniques d'embouche intensive utilisables dans les régions rizicoles.

\section{MATERIEL ET METHODE}

\section{Animaux d'expérience}

Les animaux sont des zébus Gobra âgés de 3 à 5 ans, d'un poids moyen avoisinant $250 \mathrm{~kg}$.

Six lots de 10 têtes sont constitués, cinq avec des animaux entiers, le sixième avec des animaux castrés.

\section{Alimentation}

Le facteur d'alimentation commun aux six lots est la paille de riz distribuée à volonté dans des râteliers, auquel on adjoint un concentré 
dont la composition et les modalités de distribution sont différentes pour chaque lot.

La paille de riz utilisée provient des casiers rizicoles de Richard Toll. Sa composition bromatologique moyenne est la suivante (g pour 1.000 de M.S.). Matières sèches : 919; Matières minérales: 85,7 ; Matières grasses: 17,6; Matières protéiques : 30,0 ; Matières cellulosiques : 373,1 ; Phosphore : 1,92; Calcium : 4,6 .

Les études de digestibilité in vivo réalisées sur ce fourrage au Laboratoire de Dakar lui attribuent une valeur énergétique, relativement élevée, de 0,40 à 0,46 U.F. au kg. Cependant, des observations ultérieures et les résultats obtenus par d'autres auteurs nous ont amenés à fixer cette valeur à 0,38 U.F. par $\mathrm{kg}$.

\section{Particularités des lots}

Lot 1: Animaux entiers: Dans le lot, la paille de riz est fragmentée à l'aide d'un petit hachoir électrique et on lui incorpore par mélange à la main un concentré ayant la composition suivante (concentré $n^{10} 2$ ).

Mélasse : 10; Farine de riz: 45; Son de maïs : 35; Perlurée : 4,5; Tourteau d'arachide : 0,5 ; Concentré minéral et vitaminé : 5 .

La valeur de ce concentré est estimée à 0,9 U.F. $/ \mathrm{kg}$ et $125 \mathrm{~g}$ de MAD dont $67 \mathrm{~g}$ apportés par l'urée. Les quantités distribuées au cours de l'expérience sont de $4 \mathrm{~kg}$ de paille broyée et $4 \mathrm{~kg}$ de concentré. Ce mode d'administration particulier visait à obtenir une consommation simultanée de la paille et du concentré. En fait, la stabilité du mélange s'est avérée insuffisante et les animaux ont trié d'abord le concentré pour consommer la paille broyée ensuite.

Lot 2: Il se compose d'animaux castrés qui reçoivent dans les râteliers de la paille de riz à volonté et un concentré rationné distribué dans les mangeoires.

Le concentré dans ce lot a la composition ci-après (concentré $n^{0} 1$ ).

Mélasse : 10; Farine de sorgho : 60; Gros son de blé : 10; Remoulage du blé : 8; Tourteau d'arachide : 5; Urée : 2; C.M.V. : 5.

Sa valeur alimentaire est estimée à 0,8 U.F./ $\mathrm{kg}$ et 115 MAD.
En cours d'expérience, le $25 / 5$, nous avons dû substituer la farine de maïs à la farine de sorgho en raison d'une rupture dans l'approvisionnement en cette dernière céréale, mais en fait, il s'agit d'une modification mineure.

Comme précédemment, la paille est distribuée à volonté et le concentré rationné.

Lot 3: Il est constitué par des taurillons qui reçoivent la même alimentation que le lot 2. L'intérêt de ce lot sera de permettre la comparaison des performances entre taurillons et bouvillons.

Lot 4 : Constitué de taurillons, ce lot reçoit la paille de riz à volonté et le concentré II rationné. Ses performances seront comparées à celles du lot 1 où les composants de la ration sont identiques mais les modalités de distribution différentes.

Lot 5: Les lots 5 et 6 sont particuliers. Ici, le supplément adjoint à la paille de riz vise à apporter uniquement l'azote digestible dont ce fourrage est totalement dépourvu. On escompte ainsi diminuer le prix de revient de la ration et augmenter la quantité de paille consommée. On peut supposer, en effet, que les animaux ne trouvant plus d'éléments énergétiques dans le supplément, seront contraints d'augmenter la consommation de paille pour couvrir leurs besoins.

Dans le lot 5, le supplément azoté est constitué par $1 \mathrm{~kg}$ de tourteau d'arachide et $250 \mathrm{~g}$ de C.M.V.

Dans le lot $\mathrm{n}^{\circ}$ 6, la moitié de MAD est apportée par du tourteau, l'autre moitié par de l'urée.

Ces éléments sont présents dans les proportions suivantes: Tourteau : 60; Urée: 15; C.M.V. : 25.

Des quantités suffisantes de ces suppléments azotés sont réparties chaque jour dans des mangeoires.

Durant le dernier mois de l'expérimentation et devant les faibles résultats obtenus dans ces deux derniers lots, la paille distribuée est préalablement mélassée à raison d'un $\mathrm{kg}$ de mélasse pour $6 \mathrm{~kg}$ de paille.

\section{Observation}

Ces six lots ont été soumis aux observations et mesures identiques à celles déjà décrites à 
l'occasion des expériences précédentes, ce qui nous évitera d'entrer dans leur énumération ou description; sur ces points, nous renvoyons le lecteur aux articles précédents.

\section{RESULTATS TECHNIQUES}

Ces résultats, qui sont rassemblés dans les tableaux I, II, III portent :

a) sur l'évolution pondérale dans chaque lot, d'abord au cours de chaque période, ensuite durant la totalité de l'essai;

b) sur la consommation et sur l'indice de consommation;

c) sur l'étude des carcasses.

Remarque: En début d'essai, tous les lots comportent 10 têtes. En cours d'essai et à des époques variables, sont survenus des accidents traumatiques ou pathologiques qui ont conduit à l'élimination de certains animaux.

En fin d'essai, les lots 2, 3 et 6 ne comptent plus que neuf animaux.

Tous les calculs en tiennent compte.

\section{Première période}

Elle est située entre la pesée de référence du 12 mars et celle du 9 avril 1971 et dure 28 jours.

En début d'essai, les poids moyens des lots ne sont pas significativement différents. Il en est de même à la fin de cette période.

Les gains moyens dans chaque lot, en raison de l'importance des variations à l'intérieur des lots ne sont pas non plus significativement différents $(F=2,23)$.

Le meilleur gain de poids moyen est obtenu dans le lot 3 qui reçoit le concentré riche (farine de sorgho); les plus faibles dans les lots 5 et 6 où la paille est supplémentée uniquement par du tourteau ou par le mélange tourteau/ urée.

Le broyage de la paille de riz et l'administration simultanée de la paille et du concentré n'apportent dans le lot 1 aucun bénéfice par rapport au lot 4 .

Les bouvillons du lot 2 ont un gain de poids plus faible que les taurillons du lot 3 .

Dans le lot 1 , les refus sont constitués par un mélange de paille broyée et de concentré.

TABLEAU $\mathrm{N}^{\circ} \mathrm{I}$

Performances

\begin{tabular}{|c|c|c|c|c|c|c|}
\hline Lots & 1 & 2 & 3 & 4 & 5 & 6 \\
\hline Nombre d'animaux & 10 & 9 & 9 & 10 & 10 & 9 \\
\hline Pesẻe dēbut d'essai & $271,5 \pm 20,0$ & $271,6 \pm 12,5$ & $271,9 \pm 25,7$ & $271,7 \pm 11,7$ & $270,0 \pm 15,5$ & $284,6 \pm 14,2$ \\
\hline $\begin{array}{l}\text { Gains lère période: } \\
\text { total en kg } \\
\text { par jour en g }\end{array}$ & $\begin{array}{c}20,9 \pm 5,6 \\
746\end{array}$ & $16,2 \pm 7,2$ & $\begin{array}{c}26,0 \pm 7,9 \\
928\end{array}$ & $\begin{array}{c}23,3 \pm 5,6 \\
832\end{array}$ & $\begin{array}{c}16,0 \pm 6,5 \\
571\end{array}$ & $\frac{17,4 \pm 6,1}{621}$ \\
\hline $\begin{array}{l}\text { Gains 2e période: } \\
\text { Total en kg } \\
\text { par jour en g }\end{array}$ & $\begin{array}{c}19,6 \pm 2, B \\
725\end{array}$ & $18,1 \pm 2,9$ & $\begin{array}{c}15,5 \pm 2,2 \\
574\end{array}$ & $\begin{array}{c}17,1 \pm 5,0 \\
633\end{array}$ & $\begin{array}{c}5,4 \pm 3,1 \\
199\end{array}$ & $8,5 \pm 2,9$ \\
\hline $\begin{array}{l}\text { Gains 3e pêriode: } \\
\text { total en } \mathrm{kg} \\
\text { par jour en } \mathrm{g}\end{array}$ & $\begin{array}{c}10,4 \pm 2,6 \\
693\end{array}$ & $10,5 \pm 1,8$ & $\begin{array}{c}16,4 \pm 3,4 \\
1093\end{array}$ & $\begin{array}{l}9,8 \pm 2,4 \\
653\end{array}$ & $\underset{359}{5,4 \pm 2,7}$ & $\begin{array}{c}1,10 \pm 3,3 \\
73\end{array}$ \\
\hline $\begin{array}{l}\text { Gains 5e pêriode: } \\
\text { total en } \mathrm{kg} \\
\text { par jour en } \mathrm{g}\end{array}$ & $\underset{542}{15,2 \pm 3,8}$ & $\begin{array}{c}11,13 \pm 3,2 \\
397\end{array}$ & $\begin{array}{c}16,10 \pm 3,9 \\
574\end{array}$ & $\begin{array}{c}14,4 \pm 4,8 \\
514\end{array}$ & $\begin{array}{c}10,9 \pm 3,2 \\
389\end{array}$ & $\begin{array}{r}15,5 \pm 4,1 \\
553\end{array}$ \\
\hline Poids fin d'essai & $349,0 \pm 27,5$ & $337,1 \pm 16,7$ & $354,0 \pm 31,6$ & $346,4 \pm 17,8$ & $314,4 \pm 23,1$ & $331,6 \pm 16,35$ \\
\hline $\begin{array}{l}\text { Gains totaux en kg } \\
\text { Gains par jour en } g\end{array}$ & $\begin{array}{c}77,5 \pm 11,0 \\
698\end{array}$ & $65,5 \pm 9,4$ & $\begin{array}{c}82,1 \pm 10,32 \\
739\end{array}$ & $\begin{array}{c}74,7 \pm 11,8 \\
672\end{array}$ & $\begin{array}{c}44,4 \pm 12,1 \\
399\end{array}$ & $47 \pm 10,3$ \\
\hline
\end{tabular}


TABLEAU $\mathrm{N}^{\circ}$ II

c O n s m mation

\begin{tabular}{|c|c|c|c|c|c|c|c|}
\hline Lots & & 1 & 2 & 3 & 4 & 5 & 6 \\
\hline $\begin{array}{l}\text { lère } \\
\text { période }\end{array}$ & $\begin{array}{l}\text { Paille } \\
\text { Concentré } \\
\text { Valeur UF ration } \\
\text { Indice de } \\
\text { consomation }\end{array}$ & $\begin{array}{c}8,5 \pm 0,9 \\
4,9 \\
6,6\end{array}$ & $\begin{array}{c}5,3 \pm 0,3 \\
3,7 \pm 0,7 \\
5,0 \\
10,26\end{array}$ & {$\left[\begin{array}{cc}5,5 & \pm 0,6 \\
3,9 & \pm 0,2 \\
5,21 \\
5,61\end{array}\right.$} & $\mid \begin{aligned} 5,4 & \pm 0,3 \\
3,8 & \pm 0,5 \\
& 5,48 \\
& 6,59\end{aligned}$ & $\begin{array}{c}7,2 \pm 0,9 \\
1,00 \pm 0,12 \\
3,73 \\
6,53\end{array}$ & $\begin{array}{c}7,2 \pm 0,8 \\
0,470 \pm 0,07 \\
3,01 \\
4,85\end{array}$ \\
\hline $\begin{array}{l}2 \mathrm{e} \\
\text { pêriode }\end{array}$ & $\begin{array}{l}\text { Paille } \\
\text { Concentré } \\
\text { Valeur UF ration } \\
\text { Indice de } \\
\text { consommation }\end{array}$ & $\begin{array}{c}9,8 \pm 1,0 \\
5,9 \\
7,76\end{array}$ & $\begin{array}{c}5,4 \pm 0,2 \\
4,45 \pm 0,1 \\
5,6 \\
8,35\end{array}$ & $\left\{\begin{array}{c}5,7 \pm 0,3 \\
4,46 \pm 0,06 \\
5,7 \\
9,93\end{array}\right.$ & $\left\{\begin{array}{c}5,2 \pm 0,2 \\
4,46 \pm 0,08 \\
6,0 \\
9,47\end{array}\right.$ & $\begin{array}{c}7,4 \pm 0,2 \\
1,210 \pm 0,09 \\
3,8 \\
19,09\end{array}$ & $\begin{array}{c}7,9 \pm 0,15 \\
0,500 \pm 0,1 \\
3,3 \\
10,50\end{array}$ \\
\hline $\begin{array}{l}\text { Je } \\
\text { période }\end{array}$ & $\begin{array}{l}\text { Paille } \\
\text { Concentré } \\
\text { Yaleur UF ration } \\
\text { Indice de } \\
\text { consommation }\end{array}$ & $\begin{array}{c}9,9 \pm 0,5 \\
6,08 \\
8,77\end{array}$ & $\begin{array}{c}5,4 \pm 0,2 \\
5,0 \\
6,04 \\
8,64\end{array}$ & $\begin{array}{c}5,9 \pm 0,3 \\
5,0 \\
6,24 \\
8,64\end{array}$ & $\begin{array}{c}5,4 \pm 0,2 \\
5,0 \\
6,55 \\
10,03\end{array}$ & $\begin{array}{c}7,4 \pm 0,2 \\
1,28 \\
3,85 \\
10,72\end{array}$ & $\begin{array}{c}8,2 \pm 0,3 \\
0,700 \\
3,54 \\
48\end{array}$ \\
\hline $5 \mathrm{e}$ & $\begin{array}{l}\text { Paille } \\
\text { Concentré } \\
\text { Valeur UF ration } \\
\text { Indice de } \\
\text { consonmation }\end{array}$ & $\begin{array}{c}10,5 \pm 0,2 \\
6,7 \\
12,36\end{array}$ & $\left\{\begin{array}{c}5,0 \pm 1,1 \\
5,3 \pm 1,1 \\
6,14 \\
15,46\end{array}\right.$ & $\begin{array}{c}5,4 \pm 0,2 \\
5,3 \pm 1,1 \\
6,3 \\
10,97\end{array}$ & $\left\{\begin{array}{c}4,8 \pm 1,1 \\
5,3 \pm 1,1 \\
6,6 \\
12,84\end{array}\right.$ & $\begin{array}{r}7 \quad \pm 0,7 \\
1,300 \pm 0 \\
4,44 \\
11,41\end{array}$ & $\begin{array}{c}7,4 \pm 0,5 \\
0,800 \pm 0,3 \\
4,32 \\
7,81\end{array}$ \\
\hline Moyernes & $\begin{array}{l}\text { Paille } \\
\text { Concentré } \\
\text { Valeur UF ration } \\
\text { Indice de } \\
\text { consommation }\end{array}$ & $\begin{array}{l}10,2 \\
6,0 \\
8,59\end{array}$ & $\begin{array}{l}5,25 \\
4,56 \\
5,6 \\
9,49\end{array}$ & $\begin{array}{l}5,58 \\
4,62 \\
5,8 \\
7,84\end{array}$ & $\begin{array}{l}5,17 \\
4,59 \\
6,1 \\
9,07\end{array}$ & $\begin{array}{l}7,32 \\
1,185 \\
3,96 \\
9,92\end{array}$ & $\begin{array}{l}7,68 \\
0,607 \\
3,53 \\
8,34\end{array}$ \\
\hline
\end{tabular}

La consommation de paille est supérieure dans les lots 5 et 6 . Les animaux tendent à augmenter leur ingestion de fourrage pour compenser le déséquilibre énergie/azote de la ration. Malgré cet effort, la consommation de fourrage est insuffisante pour produire un apport énergétique aussi élevé que dans les autres lots (3,54 et 3,03 U.F.). Les indices de consommation dans ces deux derniers lots sont faibles et témoignent d'une remarquable utilisation des apports.

\section{Deuxième période}

Elle va de la pesée du 9 avril à celle du 6 mai et s'étend sur 27 jours.

\section{Tableau $\mathrm{n}^{0}$ III}

La comparaison des lots par analyse de variance donne sur les gains de poids les résultats suivants :

En ce qui concerne les gains de poids, on observe des différences entre les lots comme le révèle l'analyse de la variance portant sur l'ensemble des lots $(F-8,16)$. En fait, il existe deux groupes au comportement pondéral très différent: les quatre premiers lots d'une part, les deux derniers de l'autre. En effet, la comparaison de ces deux groupes donne à $\mathrm{F}$ une valeur très élevée (34).

Il n'existe pas de différence significative entre taurillons et bouvillons pas plus qu'il n'en existe entre les lots 1 et 4 .

Consommation: Les quantités de concentré distribuées dans les lots $1,2,3,4$ sont augmentées et passent à $4,5 \mathrm{~kg}$ par animal et par jour.

La consommation de paille dans les quatre premiers lots se maintient au même niveau.

Les concentrés donnés deux fois par jour sont consommés en totalité.

Dans les lots 5 et 6 , la consommation de paille se stabilise également: environ $2 \mathrm{~kg}$ de plus que dans les premiers lots, mais la pauvreté 
énergétique de la ration se traduit par un gain faible et un indice de consommation croissant. Il faut cependant souligner que les résultats particulièrement mauvais dans le lot 5 sont la conséquence d'une rickettsiose survenue sur un animal de ce lot. L'animal a été maintenu cependant car le traitement a assuré un prompt rétablissement.

\section{Troisième période}

Elle est plus courte que les précédentes car des adaptations de ration ont été jugées nécessaires et effectuées après une pesée de référence pratiquée le 21 mai.

Les résultats intéressent donc une période de 15 jours, du 6 mai au 21 mai.

On observe durant ces 15 jours une grande efficacité de la ration dans le lot 3 (concentré riche). Le gain de poids dans le lot 5 se rétablit légèrement, mais par contre, le lot 6 ne présente pratiquement plus aucune croissance.

L'analyse de la variance montre une différence hautement significative des gains de poids entre les lots $(F=13)$.

La différence porte essentiellement sur les deux groupes $(1,2,3,4)$ et $(5,6)$. La valeur de $\mathrm{F}$ s'élève à 12 .

Enfin, dans cette période la différence entre le lot 3 et 4 due à la nature des concentrés est très significative $(F=40)$.

Consommation: La quantité de concentré distribuée a été portée à $5 \mathrm{~kg}$ par jour et par animal.

La valeur de l'indice dans le lot 6 témoigne de la très mauvaise utilisation de la ration.

\section{Quatrième période}

Les lots 5 et 6 dont le comportement est très singulier, reçoivent indubitablement une ration insuffisante en énergie. L'augmentation de consommation de fourrage par rapport aux autres lots est insuffisante pour compenser l'absence de concentré. Pour enrichir la ration, il est alors décidé de mélasser la paille de riz, en l'arrosant et la mélangeant avec de la mélasse diluée par moitié, et à raison de $2 \mathrm{~kg}$ de mélasse concentrée pour $6 \mathrm{~kg}$ de paille. Le fourrage mélassé prend donc une valeur théorique de 0,52 U.F. au kg.
D'autre part, devant l'impossibilité de réapprovisionnement en sorgho, le concentré $\mathrm{n}^{\circ} 1$ est composé avec du maïs. En raison de ces divers changements, la $4^{\mathrm{e}}$ période allant du 21 mai au 4 juin est considérée comme un intervalle d'adaptation et ne fait pas l'objet d'une étude systématique.

\section{Cinquième période}

Il s'agit de la dernière phase de l'expérimentation qui s'étend de la pesée du 4 juin à la dernière, effectuée le 2 juillet. Elle a donc une durée de 28 jours.

Le mélassage de la paille dans les lots 5 et 6 produit un rétablissement du gain de poids sensible surtout dans le lot 6. L'énergie apportée par la mélasse permet une bonne utilisation du supplément azoté et particulièrement quand il s'agit de l'urée. Il est regrettable que ce type de ration n'ait pu être expérimenté que pendant un mois; l'épuisement du stock de paille de riz en est responsable.

Dans les autres lots, le gain diminue légèrement de façon uniforme.

Ce fléchissement lié à l'arrêt de la croissance remplacée par les dépôts de graisse s'observe assez généralement en fin des essais d'embouche.

Consommation: Les concentrés sont distribués à raison de $5,5 \mathrm{~kg}$ par jour et par animal.

Durant cette période, le gain de poids n'est à nouveau pas différent entre les lots $(F=1,6)$.

Les deux derniers lots pour lesquels l'apport d'énergie a été augmenté ne se distinguent plus des quatre autres. L'indice de consommation le plus favorable est obtenu dans le lot 6 qui associe la paille mélassée et l'urée.

\section{Performances dans chaque lot pour la totalité de l'essai}

Cette expérimentation a duré en totalité 111 jours. La ration est restée constante à l'exception du changement mineur survenu dans les lots 3 et 4 où la farine de sorgho contenue dans le concentré 1 a été remplacée par la farine de maïs.

Pour les lots 5 et 6 , le mélassage de la paille dans la dernière partie de l'essai constitue une adaptation efficace comme en témoignent les résultats. 
GRAPH 1
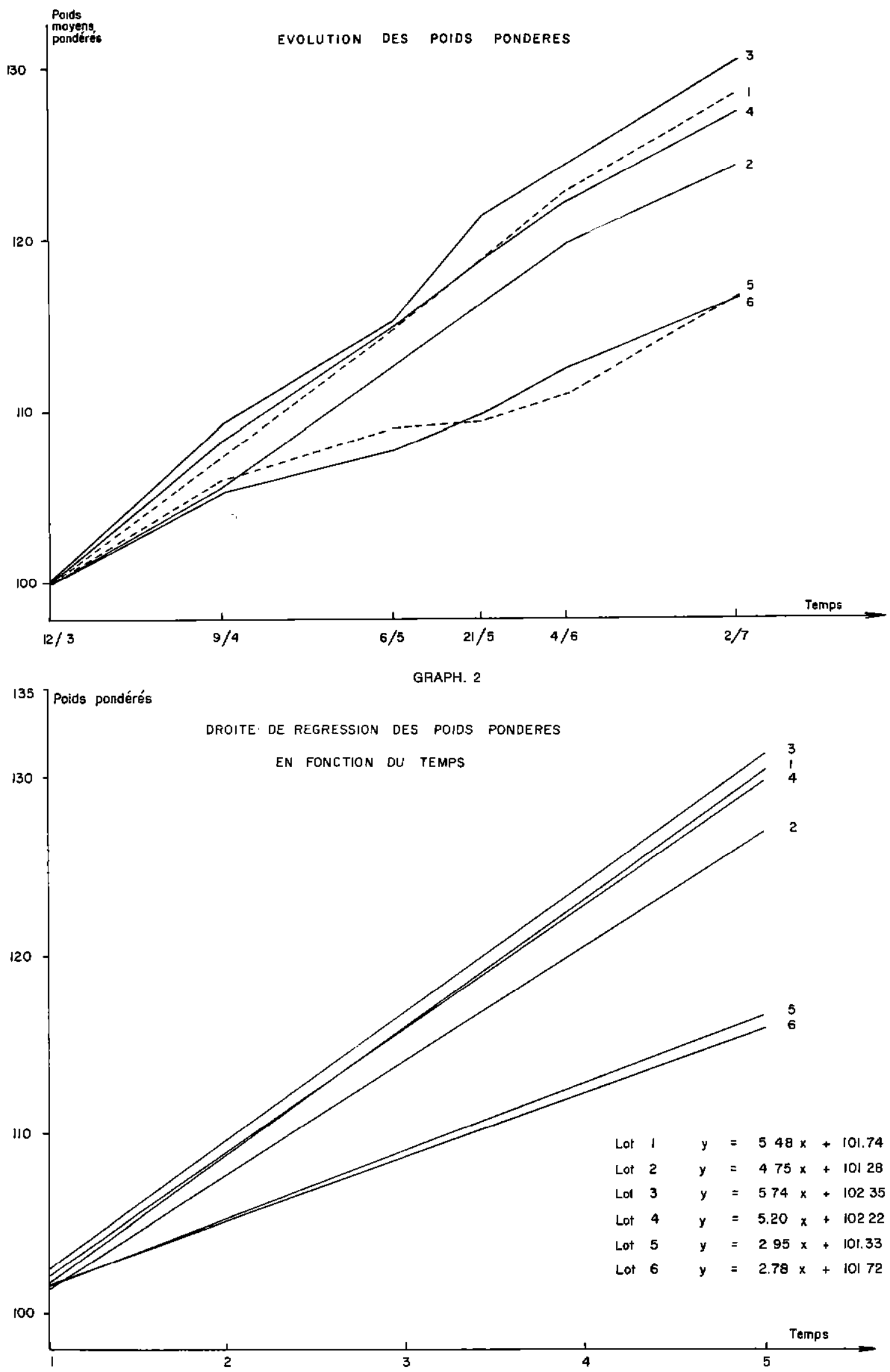
Le tableau I et le graphique résument les résultats généraux obtenus.

Pour la totalité de l'essai, le gain de poids a été le meilleur dans le lot 3 , le lot 1 vient ensuite, suivi de très près par le lot 4 . Les lots 2,6 et 5 viennent en dernier.

L'indice de consommation a été le plus faible pour le lot 3, suivi par le lot 6 .

Le prix de revient brut de la ration journalière par animal dans chaque lot est le suivant:

$$
\begin{aligned}
& \text { Lot } 1=63,45 \mathrm{~F} \mathrm{CFA} \\
& \text { Lot } 2=104,7 \text { F CFA } \\
& \text { Lot } 3=106,80 \mathrm{~F} \mathrm{CFA} \\
& \text { Lot } 4=62,0 \mathrm{~F} \mathrm{CFA} \\
& \text { Lot } 5=49,50 \mathrm{~F} \mathrm{CFA} \\
& \text { Lot } 6=39,40 \mathrm{~F} \mathrm{CFA}
\end{aligned}
$$

Le graphique 2 présente la droite de régression des poids pondérés en fonction du temps pour chaque lot.

TABLEAU No III

Poids moyens pondêrês dans chaque lot

\begin{tabular}{|c|c|c|c|c|c|}
\hline 1 & 2 & 3 & 4 & 5 & 6 \\
\hline 100 & 100 & 100 & 100 & 100 & 100 \\
\hline 107,7 & 105,9 & 109,5 & 108,5 & 105,9 & 106,11 \\
\hline 114,9 & 112,6 & 115,2 & 114,8 & 107,9 & 109,1 \\
\hline 118,7 & 116,5 & 121,2 & 118,4 & 109,9 & 109,4 \\
\hline 122,9 & 119,9 & 124,2 & 122,2 & 112,4 & 111,0 \\
\hline 128,5 & 124,1 & 130,2 & 127,5 & 116,4 & 116,5 \\
\hline
\end{tabular}

\section{Etude des carcasses}

Cinq taurillons pris au hasard sont abattus en début d'essai et servent de * témoins carcasses » qui seront comparés aux animaux abattus en fin d'embouche.

Ces derniers sont alors répartis en trois classes suivant leur gain de poids.

La classe 1 groupe les cinq animaux ayant présenté le meilleur gain de poids.

La classe 2, les cinq se rapprochant le plus du gain moyen.
La classe 3, les cinq ayant eu le gain le plus faible.

Les observations et les mesures sont celles pratiquées chaque fois dans ce type d'expérience et les résultats sont présentés dans le tableau $n^{\circ}$ IV.

La comparaison peut être effectuée entre les témoins et les résultats en fin d'essai obtenus dans la classe 2 .

Le poids moyen des carcasses chaudes en fin d'essai est de $173 \mathrm{~kg} \pm 35,91$ contre $122,3 \pm 4,8$. L'embouche a donc produit un alourdissement moyen des carcasses de plus de $50 \mathrm{~kg}$.

Le rendement après 24 heures de jeûne qui était au début de 48,5 p. 100 passe à plus de 55 p. 100 . Le poids des parties nobles, le globe par exemple, passe de 47,7 p. 100 à 49,8 p. 100 du poids de la carcasse froide alors que celui de l'épaule a tendance à diminuer $(22,9$ p. 100 à 18,7 p. 100). L'état d'engraissement en fin d'essai est amélioré comme en témoigne l'augmentation de l'indice de gras.

Indice de gras $=$

$$
\frac{\text { poids gras de rognon }}{\text { poids carcasse froide }} \times 100
$$

Celui-ci passe de 0 à 1,4 .

L'embouche a donc produit, non seulement une augmentation de la production de viande et du rendement, mais encore, une nette amélioration de la qualité.

\section{DISCUSSIONS}

Elles portent sur trois problèmes :

- la comparaison des performances dans les différents lots;

- l'esquisse financière de l'opération d'embouche avec chaque ration envisagée.

\section{Comparaison des performances dans chaque lot}

La comparaison va utiliser les pentes des droites de régression des poids en fonction du temps, droites qui constituent la meilleure approximation de l'évolution pondérale de chaque animal. 
TABLEAU $\mathbf{N}^{*}$ IV

Etudedes carcas ses

Poids avant jeĉne Poids après jeûne Pourcentage de perte au jeûne

Poids carcasses chaudes Polds carcasses froides Pourcentage de perte au ressuyuge

Rendement

\section{Rendement vra}

Pourcentage contenu

de panse

Pourcentage 5e quartier

En p.100 de carcasse

froide

- épaule

- $p$ is

- panneau

train de cote

globe

.bosse

.gras de rognon

Longueur carcasse

Epaisseur cuisse

Epaisseur plat de côte

Indice de gras

\begin{tabular}{|c|c|c|c|c|c|c|c|c|c|c|c|c|c|c|c|}
\hline \multirow{3}{*}{$\begin{array}{c}\text { Taurillons } \\
\text { témolns } \\
\overline{\mathrm{x}} \pm \text { intervalle } \\
\text { de confiance }\end{array}$} & \multicolumn{15}{|c|}{$A b a t t a g e s$ e $n$ f $i n$ d'e $s s a i$} \\
\hline & \multicolumn{3}{|c|}{ Lot 1} & \multicolumn{3}{|c|}{ Lot 2} & \multicolumn{3}{|c|}{ Lot 3} & \multicolumn{3}{|c|}{ Lot 4} & \multicolumn{3}{|c|}{ Lot 5} \\
\hline & CL. I & C1.II & C1.III & C1. I & C1.II & C1.III & C1. I & Cl.II & C1.III & C1. I & C1.II & C1.III & $\mathrm{C} 1, \mathrm{I}$ & C1.II & Cl.III \\
\hline $263,6 \pm 11,0$ & 430 & 417 & 346 & 421 & 352 & 291 & 360 & 322 & 304 & 380 & 296 & 276 & 342 & 298 & 308 \\
\hline $251,6 \pm 11,3$ & 390 & 377 & 310 & 389 & 319 & 262 & 334 & 300 & 285 & 351 & 273 & 247 & 318 & 275 & 282 \\
\hline $4,5 \pm 0,87$ & 9,3 & 9,5 & 10,4 & 7,6 & 9,3 & 9,9 & 7,2 & 6,8 & 6,2 & 7,6 & 7,7 & 10,5 & 7 & 7,7 & 8,4 \\
\hline $122,3 \pm 4,8$ & 215 & 219,4 & 174,2 & 236,2 & 186 & 144,8 & 195,5 & 171 & 170,7 & 205,2 & 143,8 & 123 & 165,1 & 144,8 & 133,4 \\
\hline $117,6 \pm 4,4$ & 212,6 & 215,1 & 169,9 & 234 & 182,8 & 141,4 & 192,2 & 166,7 & 166,3 & 202,8 & 138,4 & 118,7 & 159,7 & 139,4 & 127,1 \\
\hline $3,8 \pm 0,31$ & 1,1 & 1,01 & 1,02 & 0,93 & 1,01 & 1,02 & 1,68 & 1,02 & 1,02 & 1,16 & 1,03 & 1,03 & 3,27 & 1,03 & 1,04 \\
\hline $48,5 \pm 1,0$ & 55,12 & 58,1 & 54,8 & 60,71 & 58,3 & 55,2 & 58,53 & 57 & 59,8 & 58,46 & 52,6 & 49,7 & 51,91 & 52,6 & 47,3 \\
\hline $58,1 \pm 0,82$ & 61,25 & 64,1 & 62,5 & 65,40 & 63,5 & 62 & 63,06 & 62,7 & 63,8 & 65,06 & 60,4 & 57,3 & 61,42 & 60,7 & 55,9 \\
\hline $16,3 \pm 2,8$ & 10 & 9,6 & 10,1 & 7,9 & 8,2 & 10,9 & 7,18 & 8,9 & 6,2 & 10,14 & 12,9 & 13,2 & 15,47 & 13,3 & 15,4 \\
\hline $27,9 \pm 2,6$ & 29,1 & 27,7 & 32 & 26,8 & 33,2 & 29,3 & 25,5 & 30 & 27,8 & 27.2 & 30 & 31,9 & 30,8 & 29,9 & 31,8 \\
\hline $22,9 \pm 1,5$ & 20,4 & 19,4 & 20,7 & 21,5 & 17,4 & 19,7 & 19,7 & 19,3 & 17,8 & 19,7 & 17,4 & 20,4 & 19,5 & 20,2 & 18,7 \\
\hline $10,1 \pm 0,82$ & 10,7 & 11,2 & 9,4 & 11,7 & 10,7 & 9,6 & 10,6 & 10,6 & 12,4 & 10,3 & 9,4 & 9,01 & 9,7 & 9,5 & 10,9 \\
\hline $5,0 \pm 0,56$ & 4,8 & 3,9 & 4,1 & 4,7 & 3,3 & 3,8 & 4,1 & 3,6 & 4,5 & 4,8 & 6,2 & 4,3 & 3,6 & & 5 \\
\hline $8,8 \pm 0,31$ & 8,5 & 6,9 & 7,2 & 7,5 & 8 & 6,6 & 8,7 & 11,9 & 7,4 & 9,8 & 6,2 & 6,1 & 7,3 & 7,6 & 7,1 \\
\hline $47,7 \pm 0,59$ & 45,4 & 46,2 & 52,1 & 41,3 & 48,4 & 49,5 & 47,8 & 51,9 & 47,5 & 48,9 & 52,4 & 43,6 & 51,2 & 50,5 & 62,5 \\
\hline \multirow[t]{2}{*}{$0,6 \pm 0,20$} & 1,8 & 2,5 & 1,6 & 3,4 & 1,9 & 1,4 & 2,4 & 2,1 & 2 & 3,1 & 1,4 & 2 & 2,07 & 1 & 1,5 \\
\hline & 1,3 & 1,9 & 1 & 1,8 & 1,5 & 0,7 & 2,08 & 1,3 & 1,7 & 2,08 & 1,4 & 1,1 & 1,21 & 1 & 0,9 \\
\hline $108,1 \pm 3,10$ & 120,5 & 117,2 & 113,2 & 119,5 & 113,7 & 106 & 116,2 & 109,2 & 108,7 & 112,7 & 110 & 110,7 & 114,7 & 113,7 & 111,5 \\
\hline $19,0 \pm 2,0$ & 23,8 & 25,3 & 21,8 & 23,1 & 23,1 & 19,4 & 22,5 & 22,5 & 21,7 & 24,5 & 21,2 & 17,6 & 21,8 & 20 & 19,4 \\
\hline \multirow[t]{2}{*}{$1,7 \pm 0,26$} & 2,6 & 3,3 & 2,5 & 2,9 & 2,8 & 2,3 & 2,3 & 2,4 & 3 & 2,9 & 2,2 & 2,2 & 2,2 & 2,1 & 1,9 \\
\hline & 1,5 & 1,9 & 1 & 2,03 & 1,5 & 0,7 & 2,1 & 1,3 & 1,8 & 1,5 & 1,4 & 1,3 & 1,2 & 1 & 0,9 \\
\hline
\end{tabular}


TABLEAU $\mathrm{N}^{\circ} \mathrm{V}$

Pentes des droites de régression

\begin{tabular}{|c|c|c|c|c|c|}
\hline 1 & 2 & 3 & 4 & 5 & 6 \\
\hline 12,697 & $13,34 \mathrm{~B}$ & 20,98 & 13,182 & 13,325 & 7,30 \\
\hline 12,082 & 11,837 & 11,23 & 9,068 & 8,962 & 8,742 \\
\hline 14,862 & 16,340 & 16,68 & 12,477 & 6,851 & 2,28 \\
\hline $14,0 B 8$ & 14,605 & 15,851 & 18,84 & $3,16 B$ & 10,10 \\
\hline $17,12 \mathrm{~B}$ & 15.722 & 14,091 & $12,98 B$ & 4,065 & 9,12 \\
\hline 14,217 & 11,22 & 13,908 & 15,197 & 9,474 & 11,162 \\
\hline 8,90 & 11,211 & 17,72 & 16,768 & 9,014 & 6,317 \\
\hline 16,237 & 9,865 & 15,434 & 15,385 & 6,462 & 10,165 \\
\hline 21,148 & 12,097 & 14,52 & 17,597 & 12,214 & 7,042 \\
\hline 14,777 & & & 9,691 & 6,394 & \\
\hline $\bar{x} 14,613 \pm 2,32$ & $12,916 \pm 1,712$ & $15,60 \pm 2,06$ & $14,119 \pm 2,31$ & $7,992 \pm 2,325$ & $8,025 \pm 2,07$ \\
\hline
\end{tabular}

Les pentes dans chaque lot font l'objet du tableau $\mathrm{V}$.

L'analyse de la variance sur ces données fournit les résultats suivants :

Sur six groupes : différence hautement significative $(F=12,33)$.

Comparaison 1 et $4 \mathrm{~F}=0,11$ non significatif;

Comparaison 2 et $3 \mathrm{~F}=5,24$ significatif;

Comparaison 3 et $4 \mathrm{~F}=1,14$ non significatif;

$(6+5)(1+2+3+4) \mathrm{F}=57,8$ hautement significatif;

Comparaison 5 et $6 \mathrm{~F}=1$ non significatif.

Les conclusions sont les suivantes :

a) La préparation (broyage de la paille et incorporation du concentré 2 à la paille broyée) n'a pas apporté une amélioration du gain de poids dans le lot 1 par rapport au lot 4 , qui reçoit le même concentré et la paille en distribution séparée.

b) Le gain de poids chez les animaux castrés du lot 2 est inférieur à celui des animaux du lot 3 , tous deux recevant la même alimentation.

c) Les concentrés 1 et 2 ne produisent pas de gains différents, malgré leur prix de revient très inégal.

d) L'ensemble des lots recevant la paille plus un concentré, a un gain de poids beaucoup plus élevé que les lots recevant la paille et un simple concentré azoté ( 5 et 6 ).
Il faut souligner, cependant, que l'adaptation réalisêe dans la dernière période (mélassage de la paille) s'est révélée efficace et qu'il est fort probable que si elle avait été réalisée dès le début, ces deux derniers lots auraient eu un comportement comparable à celui des quatre premiers.

En ce qui concerne la consommation de paille de riz, on constate que les lots 5 et 6 en absorbent environ $2 \mathrm{~kg}$ de plus que les autres. Il y a donc un effort des animaux pour compenser le déficit énergétique (absence de concentré) de la ration par une consommation supérieure ne permettant pas cependant d'assurer un niveau énergétique suffisant.

Les quantités de fourrage consommées (en matière sèche) sont en moyenne de $1,55 \mathrm{~kg}$ par $100 \mathrm{~kg}$ de poids vif dans les lots $1,2,3,4$ et de $2,3 \mathrm{~kg}$ par $100 \mathrm{~kg}$ vif dans les lots 5,6 .

La consommation totale en matière sèche est en moyenne dans le premier groupe égale à $2,85 \mathrm{~kg}$, ce qui est conforme aux résultats antérieurs et de $2,5 \mathrm{~kg}$ dans le $2^{\circ}$ groupe.

Les indices de consommation restent dans tous les lots d'un niveau relativement raisonnable. Il semble que dans ce chapitre, la présentation de la ration 1 (paille broyée plus concentré incorporé) apporte une amélioration par rapport au lot 4 dont l'aliment comprend les mêmes constituants.

Le concentré à base de sorgho est celui qui entraîne l'indice le plus faible. 
Enfin, dans la supplémentation azotée, le mélange urée/tourteau paraît supérieur au tourteau seul.

\section{Esquisse économique}

Elle consistera uniquement en un bilan entre les charges fixes représentées par l'achat des animaux et le prix de revient de la nourriture d'une part et les recettes entraînées par la commercialisation des animaux.

Les bases de ces calculs sont les suivantes:

Les poids d'achat et de vente sont pour chaque lot les poids moyens en début et fin d'essai.

— Le prix d'achat est de $50 \mathrm{~F}$ le $\mathrm{kg}$ vif.
- Le prix de vente sur pied a été de $70 \mathrm{~F}$ et de $65 \mathrm{~F}$.

- Le prix des carcasses était en juillet de $170 \mathrm{~F}$ le $\mathrm{kg}$ pour les carcasses extra, de $160 \mathrm{~F}$ pour les moyennes.

Les prix des divers constituants des rations sont :

- Paille de riz : 2,50 F (frais de ramassage et bottelage).

- Paille de riz mélassée : 2,85 F/ kg.

- Concentré $\mathrm{n}^{\circ} \mathrm{II}: 10,7 \mathrm{~F}$ le $\mathrm{kg}$.

- Concentré $\mathrm{n}^{\circ} \mathrm{I}$ : 20,1 F le kg.

- Supplément azoté du lot 5: $26 \mathrm{~F}$.

- Supplément azoté du lot $6: 32,5 \mathrm{~F}$.

Les éléments de ce bilan sont présentés dans le tableau $\mathrm{n}^{\circ}$ VI.

TABLEAU $\mathrm{N}^{\circ} \mathrm{VI}$

Bilan êcononique

\begin{tabular}{|c|c|c|c|c|c|c|}
\hline $\mathrm{N}^{\circ}$ des lots & 1 & 2 & 3 & 4 & 5 & 6 \\
\hline Poids d'achat & 271 & 272 & 272 & 272 & 270 & 285 \\
\hline Prix d'achat & 13.550 & 13.600 & 13.600 & 13.600 & 13.500 & 14.250 \\
\hline Prix de revient aliment & 7.043 & 11.620 & 11.850 & 6.880 & 5.490 & 4.370 \\
\hline Charges fixes & 20.593 & 25.220 & 25.450 & 20.480 & 18.990 & 18.620 \\
\hline $\begin{array}{l}\text { Vente sur pied (1) } \\
70 \mathrm{~F} / \mathrm{kg}(1-2-3-4) \\
65 \mathrm{~F} / \mathrm{kg}(5-6)\end{array}$ & 24.400 & 23.600 & 24.800 & 24.200 & 20.410 & 21.580 \\
\hline Bilan I & 3.807 & -1.620 & -650 & +3.720 & +1.420 & +2.960 \\
\hline $\begin{array}{l}\text { Poids carcasse avec } \\
55 \mathrm{p}, 100 \text { rendement } \\
\text { Verte en carcasse (2) } \\
170 \mathrm{~F}(1-2-3-4)\end{array}$ & 190 & 185 & 195 & 190 & 130 & 180 \\
\hline $160 \mathrm{~F}(5-6)$ & 32.300 & 31.450 & 32.900 & 32.300 & 27.200 & 20.800 \\
\hline Bilan 2 & +11.707 & +6.230 & +7.450 & +11.820 & +8.210 & +2.180 \\
\hline
\end{tabular}

TABLEAU $N^{\circ} \mathrm{VII}$

Un autre élément intéressant à considérer, est le prix de revient $\mathrm{du} \mathrm{kg}$ de gain obtenu par l'alimentation intensive, dans chaque lot.

Ces données sont présentées dans le tableau $\mathrm{n}^{\star}$ VII.

Il apparaît que les lots 1, 4, 6 donnent les meilleurs résultats économiques.

Ces derniers, pour les lots 1 et 4 , tiennent à la bonne valeur alimentaire et au bas prix de la farine de riz utilisée dans le concentré 2 .
Priy de revient du $\mathrm{kg}$ de carcasse produit en cours $d$ 'embouche

\begin{tabular}{|c|c|c|c|}
\hline Lot 1 & $\begin{array}{c}\text { Prix de } \\
\text { I'aliment }\end{array}$ & $\begin{array}{c}\text { Gain de } \\
\text { poids }\end{array}$ & $\begin{array}{c}\text { Prix 1 kg } \\
\text { gain }\end{array}$ \\
\cline { 2 - 4 } Lot 2 & 11.043 & 77,5 & 90,9 \\
\hline Lot 3 & 11.850 & 82,01 & 178 \\
\hline Lot 4 & 6.880 & 74,7 & 144,4 \\
\hline Lot 5 & 5.490 & 44,4 & 92,10 \\
\hline Lot 6 & 4.370 & 47,1 & 123,6 \\
\hline
\end{tabular}


Le faible coût de l'alimentation dans le lot 6 est également responsable de la rentabilité de ce lot.

\section{Comparaison avec l'essai antérieur}

En 1970, avaient été réalisés une série d'essais d'embouche dont les résultats ont déjà fait l'objet d'une note antérieure.

Une partie du programme poursuivi à cette époque comporta un lot de taurillons exactement comparable dans les modalités expérimentales et alimentaires au lot 4 des essais actuels (tableau VIII).

TABLEAU N $\mathrm{N}^{\circ} \mathrm{VIIT}$.

Comparaison des essais 1970 et 1971

\begin{tabular}{|l|c|c|}
\hline \multirow{2}{*}{ Durée d'embouche } & Essai 1970 & Essai 1971 \\
\cline { 2 - 3 } Nombre de têtes & $126 \mathrm{j}$ & $111 \mathrm{j}$ \\
Poids moyen au début d'essai & 11 & 10 \\
Poids moyen en fin d'essai & 357 & 271 \\
Gain moyen total & 84 & 346 \\
Gain moyen journalier & 660 & 670 \\
Indice de consommation & 9,5 & 9,1 \\
\hline
\end{tabular}

Les résultats de ces deux essais sont donc très reproductibles. Cette faible variation d'un essai à l'autre montre que la nature et la composition de la ration sont, en ce qui concerne les résultats de l'embouche, les facteurs déterminants. La variabilité biologique de l'animal entre, finalement, peu en ligne de compte lorsque les essais sont effectués aux mêmes saisons.

\section{CONCLUSIONS}

La série d'essais rapportés dans cette note avait pour objet de déterminer les conditions économiques d'utilisation en alimentation intensive de deux sous-produits de la culture du riz. Dans les régions s'adonnant à cette production, la paille de riz et les farines de cône constituent les éléments essentiels à introduire dans les rations d'embouche.

Six lots ont été constitués et les gains de poids moyens obtenus qui fluctuent entre 740 et $400 \mathrm{~g}$ durant 111 jours, permettent d'envisager favorablement l'utilisation des techniques d'embouche intensive dans ces régions.

Un bilan économique sommaire tend à prouver que l'opération, favorisée cette année par le prix élevé de la viande en fin de saison sèche, peut dans la plupart des cas, être rémunératrice.

Un certain nombre de conclusions techniques ressortent de l'agencement des lots expérimentaux.

- La paille de riz est un fourrage bien appété. Cependant, les animaux restreignent sa consommation (matières sèches) à des taux inférieurs à ceux observés, avec d'autres aliments, dans ce type d'expérience (plus de $3 \mathrm{~kg}$ de matières sèches par $100 \mathrm{~kg}$ de poids vif avec les rations à la coque).

- L'adjonction de mélasse à la paille de riz n'augmente pas la consommation de fourrage mais entraîne une nette valorisation de la ration.

- L'utilisation d'une ration «all mashed » avec la paille de riz broyée n'a pas, dans les conditions de l'expérience, constitué une supériorité sur la distribution séparée de la paille et du concentré. Sans doute, cela est-il dû à une homogénéisation insuffisante, les animaux ayant pu trier le concentré qu'ils ont consommé en premier.

Il est probable que l'adjonction de mélasse en quantité suffisante aurait pu pallier cet inconvénient.

- La paille de riz administrée avec un simple supplément azoté est capable de constituer une ration économique entraînant cependant un gain de poids insuffisant pour une embouche intensive. Le mélassage de la paille expérimenté sur une trop courte période, semble relever nettement le niveau des performances, surtout dès lors que le supplément azoté comporte de l'urée.

Il nous paraît possible d'envisager que cette dernière formule est celle qui répond le mieux aux conditions d'une embouche économique.

Le bas prix des constituants de la ration semble, en effet, le premier critère à considérer. La comparaison des lots 3 et 4 en témoigne. Les performances y sont très peu différentes, 
alors que le prix de revient du concentré, deux fois plus élevé dans le lot 3, rend l'opération moins économique.
- Enfin, une fois de plus, les animaux castrés ont présenté un gain de poids moyen inférieur à celui des animaux entiers.

\section{SUMMARY}

Intensive fattening of senegalese Peulh zebu cattle with rice straw

The authors studied the nutritive value of rice straw for intensive fattening of senegalese Peulh cattle.

This is a well palatable fodder the nutritive qualities of which are increased by molasses addition.

From the economic point of view, the best formula of rice straw feeding includes molasses and a nitrogen additive with urea.

The entire cattle grew better than castrated animals.

\section{RESUMEN}

\section{Engorde intensivo con paja de arroz de cebus Peulh de Senegal}

Los autores estudiaron el valor alimenticio de la paja de arroz para el engorde intensivo de cebues Peulh de Senegal.

Es un forraje bien apetecible cuyas cualidades nutricionales se aumentan con la adición de melaza.

La formula más interesante desde el punto de vista económico comprende además la melaza, un suplemento nitrogenado con urea.

Los animales presentaron un aumento de peso superior al de los animales castrados.

\section{BIBLIOGRAPHIE}

VALENZA (J.), CALVET (H.), ORUE (J.) et WANE (A. M.). Engraissement intensif de zébus Peulh sénégalais (Gobra), I. Mâles entiers 3 à 5 ans. Rev. Elev. Méd. vét. Pays trop., 1971, 24 (1): 79-109.

VALENZA (J.), CALVET (H.), ORUE (J.) et WANE (A.M.). Engraissement intensif de zébus Peulh sénégalais (Gobra). II, Mâles castrés 7 à 10 ans.
Rev. Elev. Méd. vét. Pays trop., 1971, 24 (1): 111-24.

CALVET (H.), BOUDERGUES (R.), REMESY (C.), ARCHAMBAULT de VENCAY (J.). Recherches sur le métabolisme du rumen chez les bovins tropicaux. I. Matériel, méthodes et étude de trois fourrages utilisés au Sénégal. Rev. Elev. Méd. vét. Pays trop., 1971, 24 (2) : 287-96. 